\title{
Analysis of Speckle Reducing Filters in Ultrasound Images
}

\author{
Arvinder Kaur \\ MTech CSE \\ Dept. of Computer Science, \\ Punjabi University, \\ Patiala, India
}

\author{
Sukhjeet Kaur Ranade \\ Associate Professor \\ Dept. of Computer Science, \\ Punjabi University, \\ Patiala, India
}

\begin{abstract}
Ultrasound is a widely used medical imaging technique used for diagnostic purposes. But the major problem with these images is that they are inherently corrupted by speckle. The presence of speckle severely hampers the interpretation and analysis of medical ultrasonic images. In this paper, a comparison of various speckle reducing spatial and wavelet based methods has been carried out while de-speckling the image. These methods are evaluated and compared in terms of filter assessment parameters namely Peak Signal to Noise Ratio (PSNR), MSSIM (Mean Structural Similarity), FOM (Figure of Merit) and Method noise and consequently classified into three categoriesoutstanding, average and below average on the basis of their performance.
\end{abstract}

\section{General Terms}

Image de-noising

\section{Keywords}

Ultrasound image, Speckle noise, Filters, Wavelets

\section{INTRODUCTION}

Ultrasound imaging is a widely used medical imaging technique for diagnostic purposes. It is useful for monitoring the growth of fetus in pregnant women. It is a non-invasive technique and uses no ionizing radiation. It is portable and provides real time images. It is relatively economic as compared to other medical technologies.

However the main disadvantage of ultrasound imaging is that it is contaminated by speckle noise. Speckle noise is defined as multiplicative noise, having a granular pattern that inherently exists in and degrades the quality of the active radar, synthetic aperture radar, medical ultrasound and optical coherence tomography images. Speckle noise is generated by the constructive and destructive interference of the reflected waves from various independent scatters within a cell resolution [1]. In other words, it occurs due to random interference between the coherent returns [2]

Speckle artefact introduces fine-false structures which reduces image contrast and masks the real boundaries of the tissues under consideration. Its occurrence may substantially compromise the diagnostic effectiveness, introducing a great level of subjectivity in the interpretation of images. Therefore, it is very important to de-speckle the ultrasound images prior to analysis and processing them to reduce the risk of misdiagnose and obtaining accurate diagnosis.

Over the years, many researchers had devoted their efforts to address this issue resulting in large number of de-noising techniques. These techniques can be classified into two groups: compounding and post-processing. Compounding involves acquiring images of the same object from different view angles, assuming no changes to or motion of the object during scanning. Then take average of multiple images to generate the final image [3]. But this approach results in loss of detail in the final image as well as increased system complexity.

Post-processing techniques do not require any hardware modification in the image reconstruction system, and hence have found a growing interest. In this, images are obtained as usual and then speckle reduction filters are applied to the image to improve its quality. These can be simply classified as local adaptive filters, anisotropic filters, non-local means filters and wavelet based approaches. Lee [4], Frost [5], Kuan [6], wiener [7], median [7] and guided [8] filters are commonly used local adaptive filters with low algorithm complexity. Speckle reducing anisotropic diffusion [9] filter has good speckle suppression ability but de-noised results may create an oversmooth phenomenon. Optimized Bayesian NL-means with block selection (OBNLM) [10] is a novel de-noising algorithm with better speckle noise removing effect but it has high algorithm complexity. Wavelet-based techniques include Visu Hard Shrink [11], Visu Soft Shrink [11], Sure Shrink [12], Bayes Shrink [13], Neigh Shrink [14], Neigh Shrink SURE [15] and Wavelet and Guided filter [16] and so on. They have been widely used in speckle de-noising because they offer a simultaneous localization in time and frequency domain [17].

\subsection{Spatial Domain De-Speckling Filters}

Spatial domain techniques involve direct manipulation of image pixels encompassed by the neighbourhood of every pixel in an image [7]. Filtering creates a new pixel with coordinates equal to the coordinates of the centre of the neighbourhood, and its value is equal to the result of filtering operation. Spatial filtering can be denoted as:

$$
g(x, y)=T[f(x, y)]
$$

where $f(x, y)$ is the noisy image, $g(x, y)$ is the de-noised image, and $T$ is an operator on $f$ defined over a neighbourhood of point $(x, y)$. Typically, the neighbourhood of point $(x, y)$ is a rectangle, centred on $(x, y)$, and it is much smaller than the size of image. The filtered image is generated as the centre of the window visits each pixel in the input image.

\subsubsection{Lee filter}

Lee [4] developed a widely used local statistics filter for speckle noise reduction. It is a linear filter which tends to minimize the mean square error as

$$
x^{\prime}=x_{m}+b\left(z-x_{m}\right)
$$

where

$$
b=\operatorname{var}(x) /\left[z_{m}^{2} \sigma_{n}^{2}+\operatorname{var}(x)\right]
$$

Here $z$ represents noisy observed image, $x$ is the original signal and $n$ is the speckle noise. $x_{m}$ and $\operatorname{var}(x)$ (a priori mean and 
variance of the original signal) can be estimated by the following expressions:

$$
x_{m}=z_{m}
$$

and

$$
\operatorname{var}(x)=\left(\operatorname{var}(z)-z_{m}^{2} \sigma_{n}^{2}\right) /\left(\sigma_{n}^{2}+1\right)
$$

In order to apply the filter, one has to check if the value of $\operatorname{var}(x)$ given by eq. (6) is negative then in that case, we have a very homogeneous area, so $\operatorname{var}(x)$ is set to zero and $x$ is equal to the local mean $z_{m}$. But if the numerator of eq. (6) is very large then it is a very high contrast region (or edge is present) and $x^{\prime}=z$

\subsubsection{Frost filter}

Frost et al [5] developed a linear minimum mean square error filter which convolves the pixel values within a fixed size window with an exponential impulse response $m$ given by:

$$
m=\exp \left[-K C_{y}\left(t_{0}\right)|t|\right]
$$

where

$$
C_{y}=\sigma_{y} / \bar{y}
$$

Here $x$ be an image pixel corrupted by multiplicative noise $n$ such that $y=n x . K$ is the filter parameter, $t_{0}$ represents the location of the processed pixel and $|t|$ is the distance measured from pixel $t_{0}$.

\subsubsection{Kuan filter}

Kuan [6] also developed a local linear minimum mean square error filter under multiplicative noise. The local statistics are computed by the same expressions as with

Lee's filter.

$$
x^{\prime}=x_{m}+b\left(z-x_{m}\right)
$$

where

$$
b=\operatorname{var}(x) /\left[z_{m}^{2} \sigma_{n}^{2}+\left(1+\sigma_{n}^{2}\right) \operatorname{var}(x)\right]
$$

where $x_{m}$ and $\operatorname{var}(x)$ (a priori mean and variance of the original signal) can be estimated by Eq. (5) and (6).

\subsubsection{Wiener filter}

Wiener filter [7] is based on the least-squared principle. It is a pixel-wise adaptive method based on statistics estimated from a local neighbourhood of each pixel. It estimates the local mean and variance around each pixel as

$$
\mu=\frac{1}{N M} \sum_{n_{1}, n_{2} \epsilon \eta} a\left(n_{1}, n_{2}\right)
$$

and

$$
\sigma^{2}=\frac{1}{N M} \sum_{n_{1}, n_{2} \epsilon \eta} a^{2}\left(n_{1}, n_{2}\right)-\mu^{2}
$$

where $\eta$ is the $N \times M$ local neighborhood of each pixel in the image A. Wiener filter then uses these local statistics to create de-noised image as

$$
b\left(n_{1}, n_{2}\right)=\mu+\frac{\sigma^{2}-v^{2}}{\sigma^{2}}\left(a\left(n_{1}, n_{2}\right)-\mu\right)
$$

where $v^{2}$ is the noise variance. If the noise variance is not given, it uses the average of all the local estimated variances.

\subsubsection{Median filter}

Median filter [7] belongs to the class of non-linear filters or order-statistics filters where response is based on ordering (ranking) the pixels contained in the image area encompassed by the filter and the replacing the value of the centre pixel with the value determined by ranking result

\subsubsection{Guided filter}

Guided filter [8] is a novel explicit image filter derived from a local linear model. It considers the content of a guidance image while computing the filtering output $q$. Guidance image can be the input image itself or another different image. Guided filter behaves as an edge-preserving smoothing operator in a special case where the guidance image I is identical to the filtering input $\mathrm{p}$.

$q$ is a linear transform of $I$ in a window $w_{k}$ centred at the pixel $k$ :

$$
q_{i}=a_{k} I_{i}+b_{k}, \quad \forall i \in w_{k}
$$

where $a_{k}$ and $b_{k}$ are linear coefficients of the window $w_{k}, I$ is the guided image, $p$ is the input image and $q$ is the output image. Because pixel $i$ is involved in all the overlapping windows $w_{k}$ that covers $i$, the value of $q_{i}$ computed in different windows is averaged out to obtain final value of $q_{i}$ by the following equations:

$$
q_{i}=\frac{1}{|w|} \sum_{k, i \in w_{k}}\left(a_{k} I_{i}+b_{k}\right)=\bar{a}_{i} I_{i}+\bar{b}_{i}
$$

where $\overline{a_{i}}=\frac{1}{|w|} \sum a_{k}$ and $\overline{b_{i}}=\frac{1}{|w|} \sum b_{k}$

\subsubsection{OBNLM filter}

It is a novel method for restoring the ultrasound images. It is an adaptation of the NL-means method to de-noise ultrasound images using a Bayesian framework for the NL-means filter [10]. An empirical estimator of $\hat{v}\left(B_{i_{k}}\right)$ of a block $\left(B_{i_{k}}\right)$ can be defined as

$$
\hat{v}\left(B_{i_{k}}\right)=\frac{\sum_{j=1}^{\left|\Delta_{i_{k}}\right|} u\left(B_{j}\right) p\left(u\left(B_{i_{k}}\right) \mid u\left(B_{j}\right)\right)}{\sum_{j=1}^{\left|\Delta_{i_{k}}\right|} p\left(u\left(B_{i_{k}}\right) \mid u\left(B_{j}\right)\right)}
$$

where $p\left(u\left(B_{i_{k}}\right) \mid u\left(B_{j}\right)\right)$ denotes the probability distribution function of $u\left(B_{i_{k}}\right)$ conditionally to $u\left(B_{j}\right)$. Based on the Bayesian formulation, the Pearson distance is used to compare image patches.

\subsection{Wavelet Based De-Speckling Methods}

In addition to spatial filters, wavelet-based methods have been considered as a popular method for suppressing speckle noise from image. Logarithmic transformation is performed on the noisy image to convert multiplicative speckle noise into additive noise, followed by wavelet decomposition to concentrate the signal energy into a few large coefficients [18]. Then the noisy wavelet coefficients are modified using certain shrinkage function. This step typically involves thresholding the wavelet coefficients to remove the noise from the noisy image without affecting the significant features of an image. Finally, the de-noised image is obtained by taking the inverse wavelet transform of the processed coefficients, followed by exponential transformation.

The two general categories of thresholding are hardthresholding and soft-thresholding.

Hard Thresholding Method - All coefficients whose magnitude is greater than the selected threshold value $T$ remain as unchanged while the others are set to zero. The hardthresholding is described as

$$
(w)^{\prime}=w \text { if }(|w|>T) \text { otherwise zero }
$$


Soft Thresholding Method - Hard thresholding is discontinuous and causes ringing effect in the de-noised image. To overcome this, Donoho [19] introduced the soft thresholding method where the coefficients with magnitude greater than the threshold are shrunk towards zero after comparing them to a threshold. The Soft-thresholding function is described as

$$
(w)^{\prime}=\operatorname{sign}(w)(|w|-T) \text { if }(|w|>T) \text { otherwise } 0
$$

where $\operatorname{sign}(x)$ is the sign function of $x$.

The various threshold selections strategies are -

\subsubsection{Visu Shrink}

This is also called as the universal threshold method [11]. A threshold is given by

$$
t=\sigma \sqrt{2 \log n}
$$

where $\sigma^{2}$ is the noise variance and $n$ is the number of samples.

The noise variance $\sigma$ is estimated as $M A D(W) / 0.6745$, where $W$ represents the wavelet coefficients from sub-band $H H 1$ and $M A D$ represents median absolute deviation.

\subsubsection{Sure Shrink}

A threshold chooser based on Stein's Unbiased Risk Estimator (SURE) was proposed by Donoho and Johnstone [12] and is called as Sure Shrink. This method specifies a threshold value $t_{j}$ for each resolution level $j$ in the wavelet transform which is referred to as level dependent thresholding. The goal of Sure Shrink is to minimize the mean squared error, defined as

$$
M S E=\frac{1}{n^{2}} \sum_{x, y=1}^{n}(z(x, y)-s(x, y))^{2}
$$

where $z(x, y)$ is the estimate of the signal while $s(x, y)$ is the original signal without noise and $n$ is the size of the signal. Sure Shrink suppresses noise by thresholding the wavelet coefficients. The Sure Shrink threshold $t^{*}$ is defined as

$$
t^{*}=\min (t, \sigma \sqrt{2 \log n})
$$

where $t$ denotes the value that minimizes Stein's Unbiased Risk Estimator, $\sigma$ is the noise variance, and $n$ is the size of the signal. Sure Shrink follows the soft thresholding rule. Sure Shrink is smoothness adaptive.

\subsubsection{Bayes Shrink}

Bayes Shrink was proposed by Chang, Yu and Vetterli [13]. The goal of this method is to minimize the Bayesian risk, and hence its name, Bayes Shrink. It uses soft thresholding and is sub band-dependent. The Bayes threshold, $t_{B}$ is defined as

$$
t_{B}=\sigma^{2} / \sigma_{S}
$$

where $\sigma^{2}$ is the noise variance and $\sigma_{S}^{2}$ is the signal variance without noise. From the definition of additive noise we have

$$
w(x, y)=s(x, y)+n(x, y)
$$

Since the noise and the signal are independent of each other, it can be stated that

$$
\sigma_{w}^{2}=\sigma_{s}^{2}+\sigma^{2}
$$

$\sigma_{w}^{2}$ can be computed as shown below:

$$
\sigma_{w}^{2}=\frac{1}{n^{2}} \sum_{x, y=1}^{n} w^{2}(x, y)
$$

The variance of the signal, $\sigma_{s}^{2}$ is computed as

$$
\sigma_{s}=\sqrt{\max \left(\sigma_{w}^{2}-\sigma^{2}, 0\right)}
$$

\subsubsection{Neigh Shrink}

This wavelet-domain image thresholding scheme incorporates neighbouring coefficients, namely Neigh Shrink [14]. Neigh Shrink method thresholds the wavelet coefficients according to the magnitude of the squared sum of all the wavelet coefficients, i.e. the local energy within the neighbourhood window. The shrinkage function for Neigh Shrink of any arbitrary $3 \times 3$ window centered at $(i, j)$ is expressed as:

$$
\Gamma_{i j}=\left(1-\frac{T_{U}^{2}}{S_{i j}^{2}}\right)_{+}
$$

where, $T_{U}$ is the universal threshold and $S_{i j}^{2}$ is the squared sum of all wavelet coefficients in the respective $3 \times 3$ window given by:

$$
S_{i j}^{2}=\sum_{n=j-1}^{j+1} \sum_{m=i-1}^{i+1} Y_{m, n}^{2}
$$

Here, + sign at the end of the formula means to keep the positive values while setting it to zero when it is negative. The estimated centre wavelet coefficient $\hat{F}_{\mathrm{ij}}$ is then calculated from its noisy counterpart $Y_{i j}$ as $\hat{F}_{\mathrm{ij}}=\Gamma_{i j} Y_{i j}$

\subsubsection{Neigh Shrink SURE}

Neigh Shrink Sure [15] is an improvement over Neigh Shrink, which has disadvantage of using a non-optimal universal threshold value and the same neighbouring window size in all wavelet sub bands. Neigh Shrink Sure can determine an optimal threshold and neighbouring window size for every sub band by the Stein's unbiased risk estimate (SURE). The optimal threshold value and window size are estimated as -

$$
\left(\lambda^{S}, L^{S}\right)=\arg \min \operatorname{SURE}\left(w_{S}, \lambda, L\right)
$$

where $\lambda^{S}$ represents optimal threshold value for sub band $S, L^{S}$ represents optimal window size for sub band $S$ which minimizes $\operatorname{SURE}\left(w_{S}, \lambda, L\right)$

\subsubsection{Wavelet and guided filter}

A new de-noising method based on an improved wavelet filter and guided filter is developed to achieve speckle suppression and feature preservation [16]. It uses an improved threshold function related to the layer number of wavelet decomposition based on the universal wavelet threshold function.

$$
T_{j}=a_{j} \sigma_{n} \sqrt{2 \log M}
$$

where $j(=1,2, \ldots J)$ are the decomposition layers of wavelet transformation, $J$ denotes the largest decomposition layer. $a_{j}$ represents the adaptive parameter of the $j$ layer. The bigger decomposition layer $j$, the smaller the parameter $a_{j}$ is. $a_{j}$ is selected as $1 / \ln (j+1)$. The Bayesian maximum a posteriori estimation is applied to obtain a new wavelet shrinkage algorithm which is used to process the wavelet coefficients of the high frequency sub bands in each layer.

$\hat{g}= \begin{cases}0 & f \leq T_{j} \\ \operatorname{sign}(f) \cdot \max \left(|f|-\frac{\sigma_{n}^{2}+\sqrt{\sigma_{n}^{4}+2 \sigma_{n}^{2} \sigma_{g}^{2}}}{\sqrt{2} \sigma_{g}}, 0\right) f>T_{j}\end{cases}$

where $\hat{g}$ is the estimation of original signal $g$ and $f$ is the observed signal. The guided filter is used to filter the speckle noise in the low frequency sub-band. 


\section{PERFORMANCE EVALUATION OF DE-SPECKLING METHODS}

In order to quantitatively evaluate the de-noising methods, the performance of the noise reduction methods are measured using measures such as, peak signal-to-noise ratio (PSNR) [16], structural similarity index (SSIM) [20] and Pratt's Figure of Merit (FOM) [16]

PSNR index is defined as the ratio between the maximum possible power of signal and the power of corrupting noise that affects the fidelity of its representation. It is most easily defined via the mean squared error (MSE). Given a noise-free $M \times N$ image $X_{i j}$, and its noisy approximation $\hat{X}_{i j}$, MSE is defined as:

$$
M S E=\frac{1}{M N} \sum_{i=1}^{M} \sum_{j=1}^{N}\left(X_{i j}-\hat{X}_{i j}\right)^{2}
$$

where $M$ and $N$ represents the length and width of the twodimensional signal $X$, respectively. The PSNR is defined as:

$$
\operatorname{PSNR}(X, \hat{X})=10 \log \left(\frac{255^{2}}{M S E}\right)
$$

where $\hat{X}$ is the estimation of signal $X$.

SSIM index is a novel method for measuring the structural similarity between two images. MSSIM index is a mean SSIM index. MSSIM is given by:

$$
\begin{aligned}
& \operatorname{MSSIM}(X, Y)=\frac{1}{M} \sum_{j=1}^{M} \operatorname{SSIM}\left(x_{j}, y_{j}\right) \\
& \operatorname{SSIM}(x, y)=\frac{\left(2 \mu_{x} \mu_{y}+C_{1}\right)\left(2 \sigma_{x y}+C_{2}\right)}{\left(\mu_{x}^{2}+\mu_{y}^{2}+C_{1}\right)\left(\sigma_{x}^{2}+\sigma_{y}^{2}+C_{2}\right)}
\end{aligned}
$$

where $\mu_{x}, \mu_{y}, \sigma_{x}^{2}, \sigma_{y}^{2}$ represents the mean and variance of the reference image $X$ and its estimation $Y$ respectively. $\sigma_{x y}$ represents the covariance of $X$ and $Y . C_{1}$ and $C_{2}$ are selected as positive. The resultant MSSIM index is a decimal value between 0 and 1 , and value 1 is only reachable in case the two pictures have the same structure.

FOM can be used to objectively evaluate the performance of edge detectors, and its definition is:

$$
\operatorname{FOM}(X, \hat{X})=\frac{1}{\max \left(N_{X}, N_{\bar{X}}\right)} \sum_{i=1}^{N_{X}} \frac{1}{1+\alpha d_{i}^{2}}
$$

where $N_{X}$ and $N_{\hat{X}}$ represent the ideal and the actual detected edge pixel number, respectively. $\alpha$ is a constant (usually $\alpha=1 / 9$ ), and $d_{i}$ represents the distance of the $i^{t h}$ edge pixel to the nearest ideal edge pixel. The value range of FOM is [0 1] with 1 being the best result.

There is another parameter called Method noise, defined as the difference between the noisy image and its de-noised image. It contains the residual information of the image after the application of the de-noising method. The performance of despeckling method is good if less structural information and more noise is present in its method noise image.

\section{EXPERIMENTAL ANALYSIS}

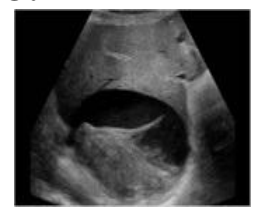

(a)

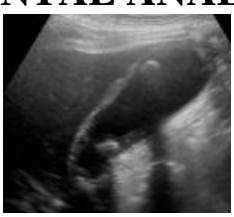

(b)

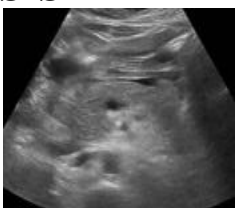

(c)

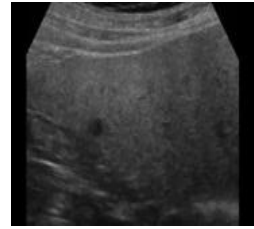

(d)

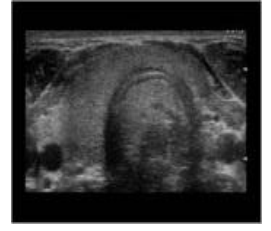

(e)
Figure 1 Noise free images of (a) Liver Cyst (b) Gallstone (c) Pancreas (d) Stomach (e) Thyroid

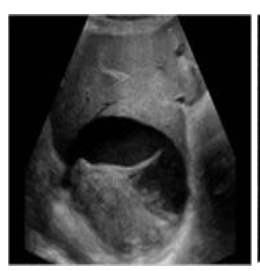

(a)

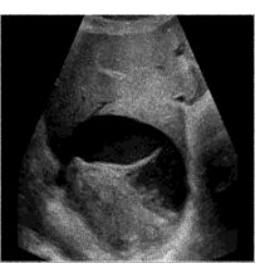

(b)

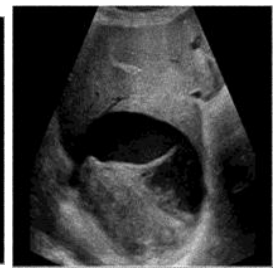

(c)

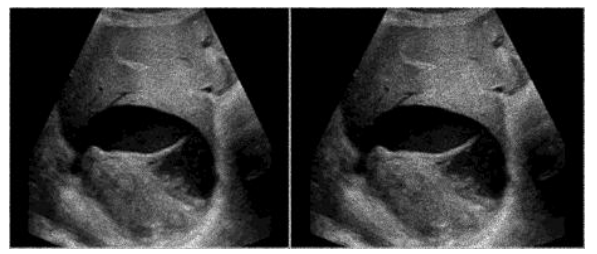

(d)

(e)

Figure 2 (a) Original Liver Cyst image (b) Noisy image with noise level 0.02 (c) Noise level 0.04 (d) Noise level 0.06 (e) Noise level 0.08

The objective of this work is to carry out a comparative evaluation of de-speckling filters on ultrasound images. An experiment is performed to compare 14 de-speckling filters (including both spatial and wavelet domain filters) and to classify them into three categories of 'Outstanding', 'Average' and 'Below average'. These methods are evaluated in terms of above filter assessment parameters. Filters used in the experiment are Lee, Frost, Kuan, Median, Wiener, OBNLM, Guided, VisuHard Shrink, VisuSoft Shrink, Sure Shrink, Bayes Shrink, Neigh Shrink, Neigh Shrink SURE and Wavelet \& Guided filter. The parameters used in various filters are determined empirically for optimal results. In this experiment, all algorithms are implemented and run on Matlab R2010a. All the wavelet based methods has used the Daubechies 4 wavelet basis with two level of decomposition of DWT.

Experiment is performed on the five images shown in Figure 1 taken from [21]. Speckle noise is added to these images with matlab function 'imnoise' at different noise levels $(=0.02,0.04$, $0.06,0.08)$. Then the abovementioned filters are applied and results are obtained for PSNR, MSSIM and FOM. The PSNR, MSSIM and FOM results for the five images at different noise levels are averaged out and then used for classification.

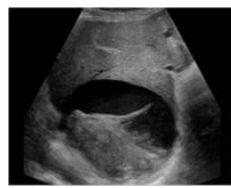

(a)

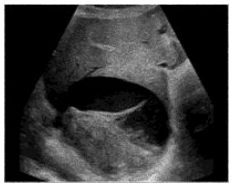

(b)

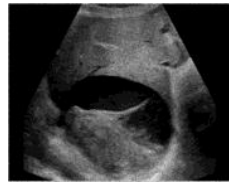

(c) 


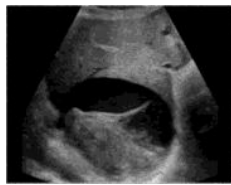

(d)

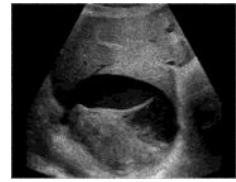

(g)

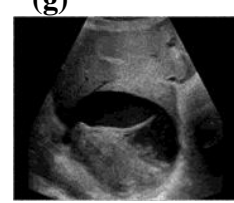

(j)

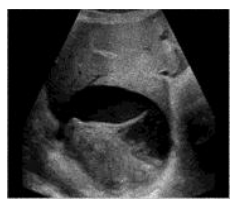

(m)

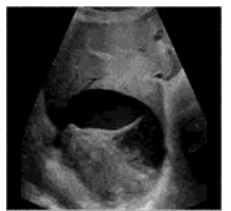

(p)

Figure 3 (a) Noise free Liver Cyst image (b) Noisy image (noise level=0.04) (c)-(p) De-noised images

In the Figure 3, (c) Lee filtered image (d) Frost filtered image (e) Kuan filtered image (f) Wiener filtered image (g) Median filtered image (h) Guided filtered image (i) OBNLM filtered image (j) Visu Hard filtered image (k) Visu Soft filtered image (l) Sure Shrink filtered image (m) Bayes Shrink filtered image (n) Neigh Shrink filtered image (o) Neigh Shrink SURE filtered image (p) Wavelet \& Guided filtered image

\subsection{Comparative analysis using PSNR -}

The average PSNR values of the five ultrasound images used in the experiment for each de-speckling filter at each noise level are tabulated in Table 1.

Based on the Average PSNR value calculated for any filter at any noise level $\mu=29.7966$ and $\sigma=1.4780$, we classify the filters into three categories as:

Below Average (B): PSNR $<\mu-\sigma$ i.e. PSNR $<28.3185$

Average (A): $\mu-\sigma \leq \mathrm{PSNR} \leq \mu+\sigma$ i.e. $28.3185 \leq \mathrm{PSNR} \leq$ 31.2796

Outstanding (O): PSNR $>\mu+\sigma$ i.e. PSNR $>31.2746$

The graph shown in Figure 4 graphically represents the results of PSNR values of different de-speckling filters when applied on ultrasound images at different speckle noise levels $(=0.02$, $0.04,0.06$ and 0.08).
The graph of Figure 5 is plotted on the basis of average PSNR values for any speckle noise level to compare the performance of 14 de-speckling filters.

Table 1 - Average PSNR of the De-noised Images

\begin{tabular}{|c|c|c|c|c|c|c|}
\hline $\begin{array}{c}\text { Noise } \\
\text { Level } \rightarrow \\
\text { Filter } \downarrow\end{array}$ & 0.02 & 0.04 & 0.06 & 0.08 & $\begin{array}{l}\text { Avg. } \\
\text { PSNR }\end{array}$ & $\begin{array}{c}\text { Cate } \\
- \\
\text { gory }\end{array}$ \\
\hline $\begin{array}{c}\text { Lee } \\
\text { Filter }\end{array}$ & $\begin{array}{c}33.090 \\
5\end{array}$ & $\begin{array}{c}30.448 \\
3\end{array}$ & $\begin{array}{c}29.033 \\
4\end{array}$ & $\begin{array}{c}28.061 \\
8\end{array}$ & $\begin{array}{c}30.158 \\
5\end{array}$ & A \\
\hline $\begin{array}{l}\text { Frost } \\
\text { filter }\end{array}$ & $\begin{array}{c}32.490 \\
1\end{array}$ & $\begin{array}{c}31.863 \\
3\end{array}$ & $\begin{array}{c}31.345 \\
1\end{array}$ & $\begin{array}{c}30.859 \\
5\end{array}$ & $\begin{array}{c}31.639 \\
5\end{array}$ & O \\
\hline $\begin{array}{l}\text { Kuan } \\
\text { Filter }\end{array}$ & $\begin{array}{c}33.133 \\
4\end{array}$ & $\begin{array}{c}30.528 \\
2\end{array}$ & $\begin{array}{c}29.142 \\
3\end{array}$ & $\begin{array}{c}28.195 \\
1\end{array}$ & $\begin{array}{c}30.249 \\
7\end{array}$ & A \\
\hline $\begin{array}{l}\text { Wiener } \\
\text { filter }\end{array}$ & $\begin{array}{c}31.992 \\
7\end{array}$ & $\begin{array}{c}28.842 \\
0\end{array}$ & $\begin{array}{c}27.085 \\
9\end{array}$ & $\begin{array}{c}25.893 \\
3\end{array}$ & $\begin{array}{c}28.453 \\
4\end{array}$ & A \\
\hline $\begin{array}{l}\text { Median } \\
\text { filter }\end{array}$ & $\begin{array}{c}32.124 \\
5\end{array}$ & $\begin{array}{c}29.508 \\
7\end{array}$ & $\begin{array}{c}27.980 \\
9\end{array}$ & $\begin{array}{c}26.816 \\
5\end{array}$ & $\begin{array}{c}29.107 \\
6\end{array}$ & A \\
\hline $\begin{array}{l}\text { Guided } \\
\text { filter }\end{array}$ & $\begin{array}{c}32.837 \\
2\end{array}$ & $\begin{array}{c}31.533 \\
0\end{array}$ & $\begin{array}{c}30.117 \\
7\end{array}$ & $\begin{array}{c}28.795 \\
9\end{array}$ & $\begin{array}{c}30.820 \\
9\end{array}$ & A \\
\hline $\begin{array}{l}\text { OBNLM } \\
\text { filter }\end{array}$ & $\begin{array}{c}32.058 \\
3\end{array}$ & $\begin{array}{c}29.844 \\
8\end{array}$ & $\begin{array}{c}27.991 \\
6\end{array}$ & $\begin{array}{c}26.383 \\
8\end{array}$ & $\begin{array}{c}29.069 \\
6\end{array}$ & A \\
\hline $\begin{array}{l}\text { VisuHar } \\
\text { d Shrink }\end{array}$ & $\begin{array}{c}31.130 \\
4\end{array}$ & $\begin{array}{c}30.316 \\
3\end{array}$ & $\begin{array}{c}29.491 \\
2\end{array}$ & $\begin{array}{c}28.712 \\
1\end{array}$ & $\begin{array}{c}29.912 \\
5\end{array}$ & A \\
\hline $\begin{array}{l}\text { VisuSoft } \\
\text { Shrink }\end{array}$ & $\begin{array}{c}33.531 \\
3\end{array}$ & $\begin{array}{c}31.294 \\
9\end{array}$ & $\begin{array}{c}29.845 \\
0\end{array}$ & $\begin{array}{c}28.691 \\
1\end{array}$ & $\begin{array}{c}30.840 \\
5\end{array}$ & A \\
\hline $\begin{array}{c}\text { Sure } \\
\text { Shrink }\end{array}$ & $\begin{array}{c}33.531 \\
3\end{array}$ & $\begin{array}{c}31.418 \\
7\end{array}$ & $\begin{array}{c}30.254 \\
1\end{array}$ & $\begin{array}{c}29.272 \\
4\end{array}$ & $\begin{array}{c}31.119 \\
1\end{array}$ & A \\
\hline $\begin{array}{l}\text { Bayes } \\
\text { Shrink }\end{array}$ & $\begin{array}{c}28.570 \\
6\end{array}$ & $\begin{array}{c}26.945 \\
8\end{array}$ & $\begin{array}{c}26.295 \\
4\end{array}$ & $\begin{array}{c}25.990 \\
0\end{array}$ & $\begin{array}{c}26.950 \\
4\end{array}$ & B \\
\hline $\begin{array}{l}\text { Neigh } \\
\text { Shrink }\end{array}$ & $\begin{array}{c}31.187 \\
3\end{array}$ & $\begin{array}{c}30.635 \\
7\end{array}$ & $\begin{array}{c}30.080 \\
1\end{array}$ & $\begin{array}{c}29.376 \\
7\end{array}$ & $\begin{array}{c}30.319 \\
9\end{array}$ & A \\
\hline $\begin{array}{l}\text { Neigh } \\
\text { Shrink } \\
\text { SURE }\end{array}$ & $\begin{array}{c}28.955 \\
5\end{array}$ & $\begin{array}{c}27.048 \\
6\end{array}$ & $\begin{array}{c}26.520 \\
4\end{array}$ & $\begin{array}{c}26.012 \\
6\end{array}$ & $\begin{array}{c}27.134 \\
2\end{array}$ & B \\
\hline $\begin{array}{l}\text { Wavelet } \\
\text { \&Guide } \\
\text { d filter }\end{array}$ & $\begin{array}{c}33.264 \\
3\end{array}$ & $\begin{array}{c}31.869 \\
7\end{array}$ & $\begin{array}{c}30.658 \\
4\end{array}$ & $\begin{array}{c}29.712 \\
0\end{array}$ & $\begin{array}{c}31.376 \\
1\end{array}$ & $\mathbf{O}$ \\
\hline $\begin{array}{l}\text { Average } \\
\text { PSNR }\end{array}$ & $\begin{array}{c}31.992 \\
6\end{array}$ & $\begin{array}{c}30.149 \\
8\end{array}$ & $\begin{array}{c}28.988 \\
6\end{array}$ & $\begin{array}{c}28.055 \\
2\end{array}$ & $\begin{array}{c}29.796 \\
6\end{array}$ & - \\
\hline
\end{tabular}

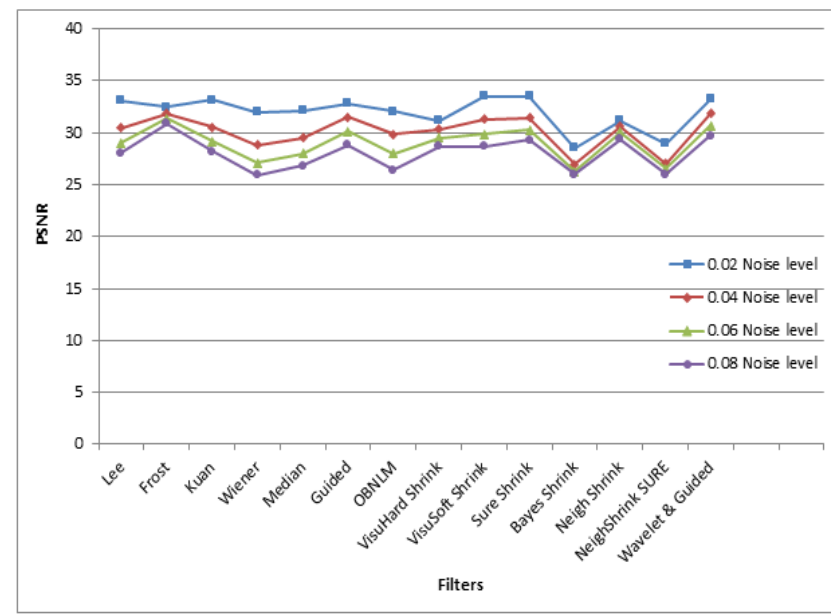

Figure 4 Graph representing PSNR values for different despeckling filters at different speckle noise levels

The graphs show that the Frost filter and Wavelet \& guided filter give higher values of average PSNR than the other filters at any level of speckle noise. But Bayes Shrink and Neigh Shrink SURE filter do not give good results in terms of PSNR. Sure Shrink filter also performs well. 


\subsection{Comparative analysis using MSSIM}

The average MSSIM values of the five ultrasound images used in the experiment for each de-speckling filter at each noise level are tabulated in Table 2.

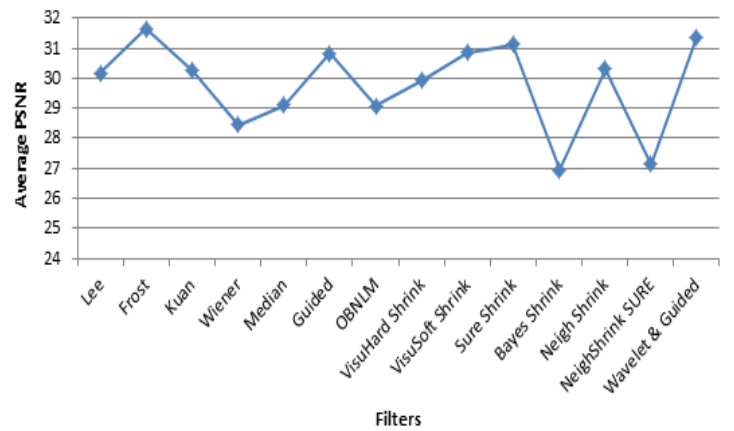

Figure 5 Graph representing average PSNR values for different de-speckling filters at any speckle noise level

Table 2 - Average MSSIM of the De-noised Images

\begin{tabular}{|c|c|c|c|c|c|c|}
\hline $\begin{array}{l}\text { Noise } \\
\text { Level } \\
\rightarrow \\
\text { Filter } \downarrow\end{array}$ & 0.02 & 0.04 & 0.06 & 0.08 & $\begin{array}{l}\text { Averag } \\
\text { e } \\
\text { MSSIM }\end{array}$ & $\begin{array}{l}\text { Cate } \\
\text {-gory }\end{array}$ \\
\hline $\begin{array}{l}\text { Lee } \\
\text { Filter }\end{array}$ & $\begin{array}{l}0.877 \\
4\end{array}$ & $\begin{array}{l}0.797 \\
3\end{array}$ & $\begin{array}{l}0.755 \\
8\end{array}$ & $\begin{array}{l}0.718 \\
5\end{array}$ & 0.7872 & A \\
\hline $\begin{array}{l}\text { Frost } \\
\text { filter }\end{array}$ & $\begin{array}{l}0.891 \\
1 \\
\end{array}$ & $\begin{array}{l}0.876 \\
6\end{array}$ & $\begin{array}{l}0.862 \\
9\end{array}$ & $\begin{array}{l}0.849 \\
9\end{array}$ & 0.8701 & O \\
\hline $\begin{array}{l}\text { Kuan } \\
\text { filter }\end{array}$ & $\begin{array}{l}0.878 \\
1\end{array}$ & $\begin{array}{l}0.806 \\
5 \\
\end{array}$ & $\begin{array}{l}0.759 \\
1 \\
\end{array}$ & $\begin{array}{l}0.723 \\
0 \\
\end{array}$ & 0.7916 & A \\
\hline $\begin{array}{l}\text { Wiener } \\
\text { filter }\end{array}$ & $\begin{array}{l}0.868 \\
4 \\
\end{array}$ & $\begin{array}{l}0.793 \\
5 \\
\end{array}$ & $\begin{array}{l}0.744 \\
5 \\
\end{array}$ & $\begin{array}{l}0.707 \\
9\end{array}$ & 0.7785 & A \\
\hline $\begin{array}{l}\text { Median } \\
\text { filter }\end{array}$ & $\begin{array}{l}0.874 \\
0\end{array}$ & $\begin{array}{l}0.796 \\
9\end{array}$ & $\begin{array}{l}0.741 \\
5\end{array}$ & $\begin{array}{l}0.697 \\
9\end{array}$ & 0.7775 & A \\
\hline $\begin{array}{l}\text { Guided } \\
\text { filter }\end{array}$ & $\begin{array}{l}0.876 \\
3 \\
\end{array}$ & $\begin{array}{l}0.845 \\
2 \\
\end{array}$ & $\begin{array}{l}0.806 \\
1\end{array}$ & $\begin{array}{l}0.767 \\
3 \\
\end{array}$ & 0.8237 & A \\
\hline $\begin{array}{l}\text { OBNL } \\
\text { M filter }\end{array}$ & $\begin{array}{l}0.881 \\
9\end{array}$ & $\begin{array}{l}0.799 \\
8 \\
\end{array}$ & $\begin{array}{l}0.726 \\
7 \\
\end{array}$ & $\begin{array}{l}0.667 \\
2\end{array}$ & 0.7689 & A \\
\hline $\begin{array}{l}\text { Visu } \\
\text { Hard } \\
\text { Shrink }\end{array}$ & $\begin{array}{l}0.826 \\
4\end{array}$ & $\begin{array}{l}0.830 \\
0\end{array}$ & $\begin{array}{l}0.820 \\
7\end{array}$ & $\begin{array}{l}0.803 \\
4\end{array}$ & 0.8201 & A \\
\hline $\begin{array}{l}\text { Visu } \\
\text { Soft } \\
\text { Shrink } \\
\end{array}$ & $\begin{array}{l}0.892 \\
5\end{array}$ & $\begin{array}{l}0.859 \\
5\end{array}$ & $\begin{array}{l}0.835 \\
7\end{array}$ & $\begin{array}{l}0.813 \\
6\end{array}$ & 0.8503 & A \\
\hline $\begin{array}{l}\text { Sure } \\
\text { Shrink }\end{array}$ & $\begin{array}{l}0.892 \\
5\end{array}$ & $\begin{array}{l}0.862 \\
2\end{array}$ & $\begin{array}{l}0.841 \\
4\end{array}$ & $\begin{array}{l}0.817 \\
4\end{array}$ & 0.8533 & A \\
\hline $\begin{array}{l}\text { Bayes } \\
\text { Shrink }\end{array}$ & $\begin{array}{l}0.742 \\
5\end{array}$ & $\begin{array}{l}0.681 \\
3\end{array}$ & $\begin{array}{l}0.658 \\
1\end{array}$ & $\begin{array}{l}0.648 \\
1\end{array}$ & 0.6825 & B \\
\hline $\begin{array}{l}\text { Neigh } \\
\text { Shrink }\end{array}$ & $\begin{array}{l}0.824 \\
3\end{array}$ & $\begin{array}{l}0.812 \\
5\end{array}$ & $\begin{array}{l}0.806 \\
9\end{array}$ & $\begin{array}{l}0.796 \\
7\end{array}$ & 0.8101 & A \\
\hline $\begin{array}{l}\text { Neigh } \\
\text { Shrink } \\
\text { SURE }\end{array}$ & $\begin{array}{l}0.756 \\
7\end{array}$ & $\begin{array}{l}0.694 \\
1\end{array}$ & $\begin{array}{l}0.665 \\
6\end{array}$ & $\begin{array}{l}0.642 \\
9\end{array}$ & 0.6898 & B \\
\hline $\begin{array}{l}\text { Wavelet } \\
\text { \& } \\
\text { Guided } \\
\text { filter }\end{array}$ & $\begin{array}{l}0.880 \\
5\end{array}$ & $\begin{array}{l}0.866 \\
2\end{array}$ & $\begin{array}{l}0.847 \\
8\end{array}$ & $\begin{array}{l}0.828 \\
5\end{array}$ & 0.8557 & O \\
\hline $\begin{array}{l}\text { Average } \\
\text { MSSIM }\end{array}$ & $\begin{array}{l}0.854 \\
4\end{array}$ & $\begin{array}{l}0.808 \\
6\end{array}$ & $\begin{array}{l}0.776 \\
6\end{array}$ & $\begin{array}{l}0.748 \\
7\end{array}$ & 0.7971 & - \\
\hline
\end{tabular}

Based on the Average MSSIM value calculated for any filter at any noise level $\mu=0.7971$ and $\sigma=0.0571$, we classify the filters into three categories as:

Below Average (B): MSSIM $<\mu-\sigma$ i.e. MSSIM $<0.7399$

Average (A): $\mu-\sigma \leq$ MSSIM $\leq \mu+\sigma$ i.e. $0.7399 \leq$ MSSIM $\leq 0.8542$
Outstanding (O): MSSIM $>\mu+\sigma$ i.e. $\operatorname{MSSIM}>0.8542$

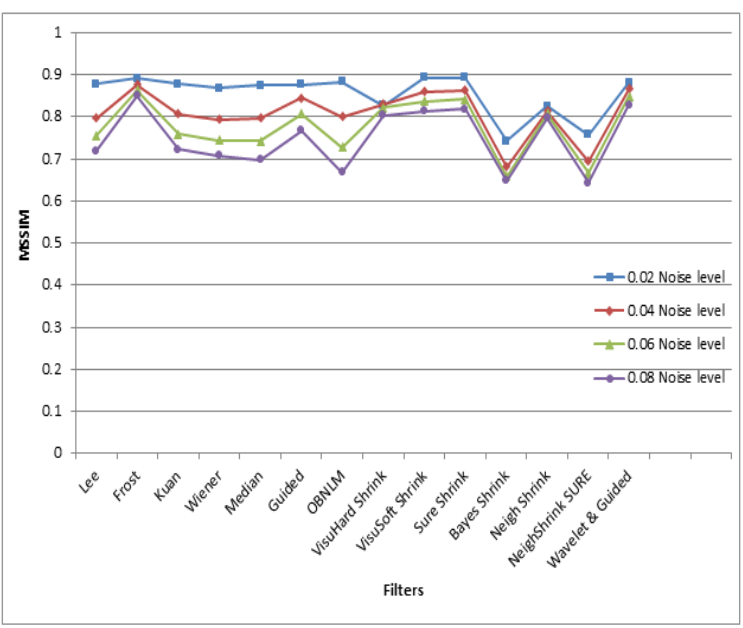

Figure 6 Graph representing MSSIM values for different de-speckling filters at different speckle noise levels

The graph shown in Figure 6 graphically represents the results of MSSIM values of different de-speckling filters when applied on ultrasound images at different speckle noise levels $(=0.02$, $0.04,0.06$ and 0.08 )

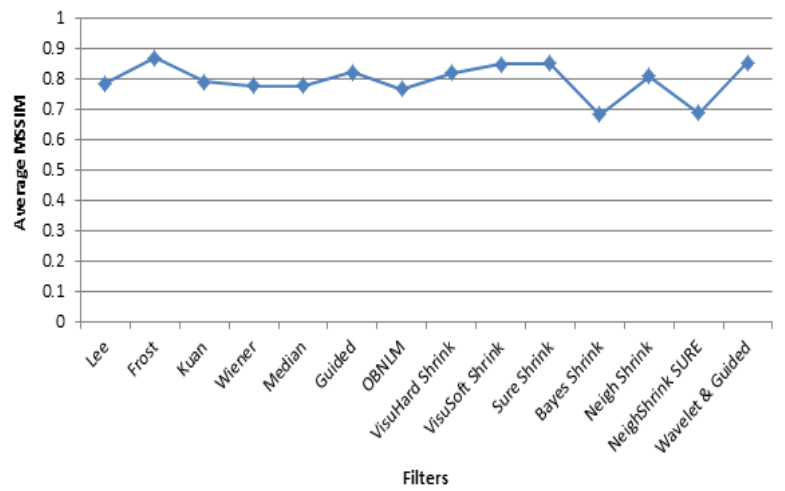

Figure 7 Graph representing average MSSIM values for different de-speckling filters at any speckle noise level

The graph in Figure 7 is plotted on the basis of average MSSIM values for any speckle noise level.

The above graphs indicate that average MSSIM values of the Frost filter and Wavelet \& guided filter for any speckle noise level are higher than the other de-speckling filters. Like PSNR, the MSSIM values of Bayes Shrink and Neigh Shrink SURE are minimum among all the filters.

\subsection{Comparative analysis using FOM -}

The average FOM values of the five ultrasound images used in the experiment for each de-speckling filter at each noise level are tabulated in Table 3.

Based on the Average FOM value (for any filter at any noise level) $\mu=0.7854$ and $\sigma=0.0324$ calculated from Table 3, we classify the filters into three categories as:

Below Average (B): FOM $<\mu-\sigma$ i.e. FOM $<0.7529$

Average (A): $\mu-\sigma \leq \mathrm{FOM} \leq \mu+\sigma$ i.e. $0.7529 \leq \mathrm{FOM} \leq$ 0.8178

Outstanding $(\mathrm{O}): \mathrm{FOM}>\mu+\sigma$ i.e. $\mathrm{FOM}>0.8178$ 
Table 3 - Average FOM of the De-noised Images

\begin{tabular}{|l|l|l|l|l|l|l|}
\hline $\begin{array}{l}\text { Noise Level } \\
\text { Filter } \downarrow\end{array}$ & $\mathbf{0 . 0 2}$ & $\mathbf{0 . 0 4}$ & $\mathbf{0 . 0 6}$ & $\mathbf{0 . 0 8}$ & $\begin{array}{l}\text { Avg. } \\
\text { FOM }\end{array}$ & $\begin{array}{l}\text { Cate- } \\
\text { gory }\end{array}$ \\
\hline Lee filter & 0.8437 & 0.7705 & 0.7384 & 0.7234 & 0.7690 & A \\
\hline Frost filter & 0.8126 & 0.8238 & 0.8283 & 0.8173 & 0.8205 & O \\
\hline Kuan filter & 0.8444 & 0.7685 & 0.7405 & 0.7234 & 0.7692 & A \\
\hline Wiener filter & 0.8362 & 0.7765 & 0.7526 & 0.7247 & 0.7725 & A \\
\hline Median filter & 0.8157 & 0.7683 & 0.7265 & 0.6766 & 0.7467 & B \\
\hline Guided filter & 0.7360 & 0.7621 & 0.7782 & 0.7650 & 0.7603 & A \\
\hline $\begin{array}{l}\text { OBNLM } \\
\text { filter }\end{array}$ & 0.7696 & 0.7625 & 0.7962 & 0.7738 & 0.7755 & A \\
\hline $\begin{array}{l}\text { Visu Hard } \\
\text { Shrink }\end{array}$ & 0.8321 & 0.8229 & 0.8197 & 0.8044 & 0.8197 & O \\
\hline $\begin{array}{l}\text { Visu Soft } \\
\text { Shrink }\end{array}$ & 0.8422 & 0.8172 & 0.8027 & 0.7959 & 0.8145 & A \\
\hline Sure Shrink & 0.8422 & 0.8187 & 0.8090 & 0.798 & 0.8169 & A \\
\hline Bayes Shrink & 0.7661 & 0.7275 & 0.7267 & 0.7145 & 0.7337 & B \\
\hline $\begin{array}{l}\text { Neigh } \\
\text { Shrink }\end{array}$ & 0.8271 & 0.8346 & 0.8217 & 0.8034 & 0.8217 & O \\
\hline $\begin{array}{l}\text { Neigh Shrink } \\
\text { SURE }\end{array}$ & 0.7811 & 0.7581 & 0.7425 & 0.7267 & 0.7521 & B \\
\hline $\begin{array}{l}\text { Wavelet \& } \\
\text { Guided } \\
\text { filter }\end{array}$ & 0.8396 & 0.8289 & 0.8186 & 0.8036 & 0.8226 & $\mathbf{O}$ \\
\hline $\begin{array}{l}\text { Average } \\
\text { FOM }\end{array}$ & 0.8134 & 0.7885 & 0.7786 & 0.7607 & $\mathbf{0 . 7 8 5 4}$ & - \\
\hline
\end{tabular}

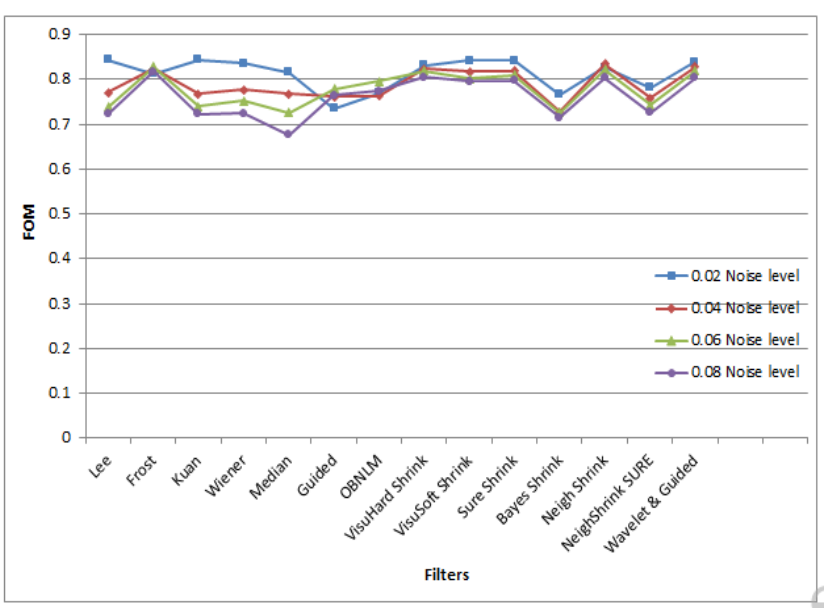

Figure 8 Graph representing FOM values for different despeckling filters at different speckle noise levels

The graph shown in Figure 8 graphically represents the results of FOM values of different de-speckling filters when applied on ultrasound images at different speckle noise levels $(=0.02,0.04$, 0.06 and 0.08 ).

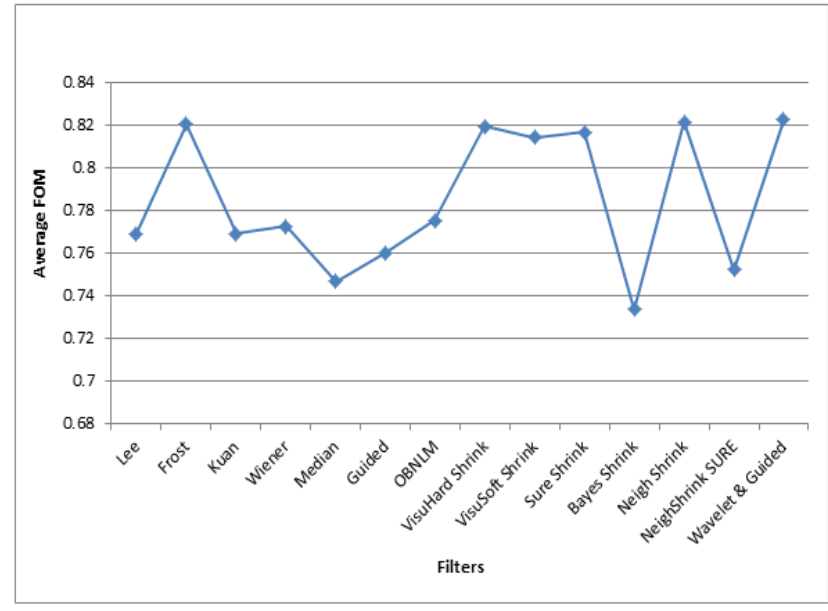

Figure 9 Graph representing average FOM values for differentde-speckling filters at any speckle noise level

The graph shown in Figure 9 is plotted on the basis of average FOM values for any speckle noise level to compare the performance of 14 de-speckling filters.

The above graphs show that FOM values of Frost filter, Visu Hard Shrink, Neigh Shrink and Wavelet \& guided filter are higher than the FOM values of other filters whereas Median, Bayes Shrink and Neigh Shrink SURE have lowest values of FOM parameter.

\subsection{Comparative analysis using Method noise}

Figure 10 shows the images of method noise obtained after despeckling the noisy liver cyst image having 0.04 speckle noise level with all the filters used in the experiment for the purpose of comparative analysis.

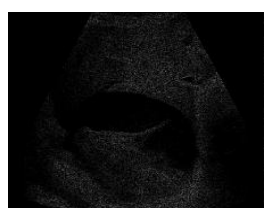

(a)

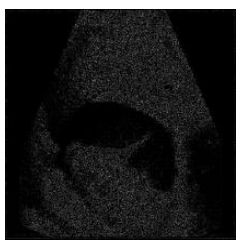

(d)

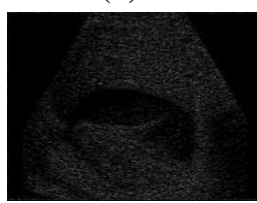

$(\mathrm{g})$

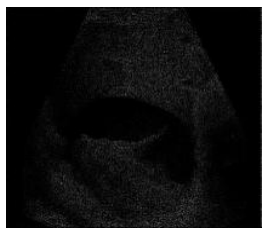

(j)

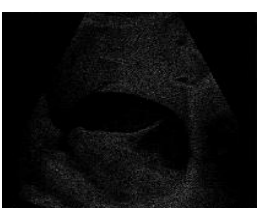

(b)

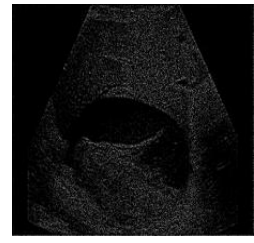

(e)

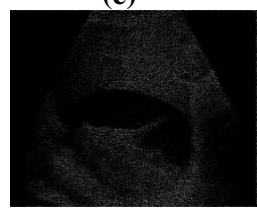

(h)

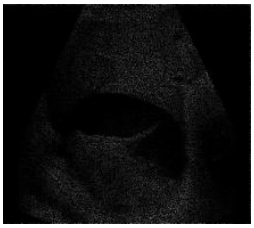

(k)

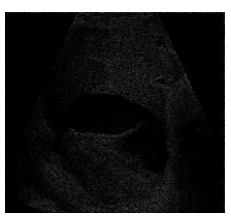

(c)

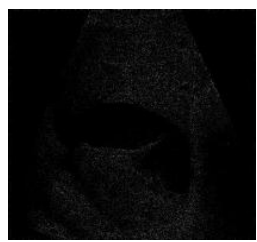

(f)

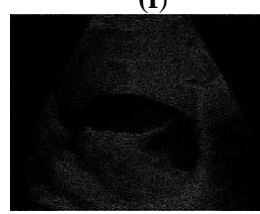

(i)

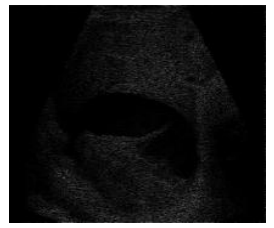

(I) 


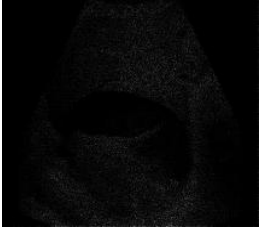

(m)

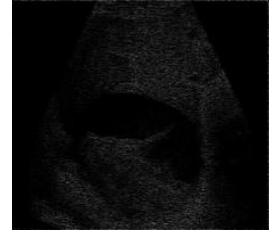

(n)
Figure 10 (a)-(n) Method noise images of liver cyst image with 0.04 noise level

In the Figure 10, Method noise images are obtained after application of (a) Lee filter (b) Frost filter (c) Kuan filter (d) Wiener filter (e) Median filter (f) Guided filter (g) OBNLM filter (h) Visu Hard filter (i) Visu Soft filter (j) Sure Shrink filter (k) Bayes Shrink filter (l) Neigh Shrink filter (m) Neigh Shrink SURE filter (n) Wavelet \& Guided filter

It follows that the performance of Median, Bayes Shrink and Neigh Shrink SURE methods is poor in suppressing speckle as their method noise images contain very little noise. Method noise image of Guided filters contains speckle noise but structural information is also present in it whereas method noise images of Wiener and OBNLM filters contain lot of noise and less structural details.

\section{CONCLUSION}

In this paper, an extensive comparative analysis of different despeckling methods including both spatial filters and wavelet methods has been carried out. Filtering is done by seven different spatial filters- Lee, Frost, Kuan, Wiener, Median, Guided and OBNLM filters and seven different wavelet thresholding methods - Visu Hard Shrink, Visu Soft Shrink, Sure Shrink Universal, Bayes Shrink, Neigh Shrink, Neigh Shrink SURE and Wavelet \& Guided filter and their results for filter assessment parameters are shown in tables and graphs. The quality of de-noised image is high if it has higher value of PSNR, MSSIM and FOM. The analysis concludes that Wavelet $\&$ Guided filter and Frost filter have higher PSNR, MSSIM and FOM at different speckle noise level and are classified as Outstanding filters whereas Bayes Shrink and Neigh Shrink SURE filters are categorised as Below Average filters as they do not give satisfactory results. Lee, Kuan, Wiener, Median, Guided, OBNLM filter, Visu Hard Shrink, Visu Soft Shrink, Sure Shrink and Neigh Shrink filters are placed in the category of Average filters.

\section{REFERENCES}

[1] N.Gupta, M.N.S. Swamy, E.Plotkin, "Despeckling of medical ultrasound images using data and rate adaptive lossy compression", IEEE Trans. Med. Ima. 24(6)(2005) 743-754.

[2] C. Saxena and D. Kourav, "Noises and Image Denoising Techniques: Brief Survey", International Journal of Emerging Technology and Advanced Engineering, Vol. 4, Issue 3, 2014

[3] M. A. Gungor and I. Karagoz, "The homogeneity map method for speckle reduction in diagnostic ultrasound images", Measurement 68(2015) 100-110
[4] J.S. Lee, "Digital image enhancement and noise filtering by use of local statistics", IEEE Trans. Pattern Anal. Mach. Intell. PAMI-2 (1980) 165-168.

[5] V.S. Frost, J.A. Stiles, K.S. Shanmugan, J.C. Holtzman, “A model for radar images and its application to adaptive digital filtering of multiplicative noise", IEEE Trans. Pattern Anal. Mach. Intell. 4 (2) (1982) 157-166.

[6] D. Kuan, A. Sawchuk, T. Strand, P. Chavel, "Adaptive Noise Smoothing Filter for Images with Signal-Dependent Noise", IEEE Trans. on Pattern Analysis and Machine Intelligence 7 (2) (1985) 165-177.

[7] R. C. Gonzalez and R. E Woods., Digital Image Processing, Pearson Education, Second Edition, 2005

[8] K. He, J. Sun, X. Tang, "Guided image filtering”, in: Proceedings of $11^{\text {th }}$ European Conference on Computer Vision, Berlin, 2010, pp. 1-14.

[9] Y. Yu, S.T. Acton, "Speckle reducing anisotropic diffusion", IEEE Trans. Image Process. 11 (11) (2002) $1260-1270$

[10] P. Coupe, P. Hellier, C. Kervrann, C. Barillot,"Nonlocal means-based speckle filtering for ultrasound images", IEEE Trans. Image Process. 18 (10) (2009) 2221-2229.

[11] D.L. Donoho, I.M. Johnstone, "Ideal spatial adaptation by wavelet shrinkage", Biometrika 81 (3) (1994) 425-455.

[12] D.L. Donoho, J.M. Johnstone, "Adapting to unknown smoothness via wavelet shrinkage", J. Am. Statist. Assoc. 90 (432) (1995) 1200-1224.

[13] S.G. Chang, B. Yu, M. Vetterli,“Adaptive wavelet thresholding for image denoising and compression", IEEE Trans. Image Process. 9 (9) (2000) 1532-1546.

[14] G.Y. Chen, T.D. Bui, A. Krzyzak, "Image denoising using neighbouring wavelet coefficients", IEEE Trans. Signal Process. 10(10)(2004) 917-920.

[15] Z. Dengwen, C. Wengang, "Image denoising with an optimal threshold and neighboring window", Pattern Recognition Letters, 29(2008) 1694-1697.

[16] J. Zhang, G. Lin, L. Wu, Y. Cheng, "Speckle filtering of medical ultrasonic images using wavelet and guided filter", Ultrasonics 65 (2016) 177-193.

[17] M. Sifuzzaman, M.R. Islam and M.Z. Ali, "Application of Wavelet Transform and its Advantages compared to Fourier Transform", Journal of Physical Sciences, Vol. 13, 2009,121-134.

[18] J. Tian and L. Chen, "Image despeckling using a nonparametric statistical model of wavelet coefficients", Biomedical Signal Processing and Control 6 (2011) 432437 [19] D. L. Donoho, "De-noising by soft-thresholding", IEEE Trans. On Information Theory, Vol 41, No. 3, May 1995.

[20] Z. Wang, A.C. Bovik, H.R. Sheikh, "Image quality accessment: from eror visibility to structural similarity", IEEE Trans. Image Process. 13(4) (2004) 600-612.

[21] www.ultrasoundcases.info 